\title{
Nitrous oxide/oxygen plus acetaminophen versus morphine in ST elevation myocardial infarction: open-label, cluster-randomized, non-inferiority study
}

Sandrine Charpentier ${ }^{1,2^{*}}$, Michel Galinski ${ }^{3}$, Vincent Bounes ${ }^{4}$, Agnès Ricard-Hibon ${ }^{5}$, Carlos El-Khoury ${ }^{6,7}$, Meyer Elbaz ${ }^{8}$, François-Xavier Ageron ${ }^{9}$, Stéphane Manzo-Silberman ${ }^{10,11}$, Louis Soulat ${ }^{12}$, Frédéric Lapostolle ${ }^{13}$, Alexandre Gérard ${ }^{14}$, Delphine Bregeaud ${ }^{15}$, Vanina Bongard ${ }^{16,17}$, Eric Bonnefoy-Cudraz ${ }^{18,19}$ and for the SCADOL II investigators

\begin{abstract}
Background: Studies have shown disparate results on the consequences of morphine use in ST-segment elevation myocardial infarction (STEMI). No study has evaluated alternative treatments that could be at least non-inferior to morphine without its potentially damaging consequences for myocardial function and platelet reactivity. The aim of this study was to evaluate whether nitrous oxide/oxygen plus intravenous acetaminophen (NOO-A) is non-inferior to morphine to control chest pain in STEMI patients.

Methods: This multicenter, open-label, cluster-randomized, controlled, non-inferiority study compared NOO-A with morphine in 684 prehospital patients with ongoing suspected STEMI of $<12 \mathrm{~h}$ duration and a pain rating score $\geq 4$. The primary endpoint was the proportion of patients achieving pain relief (numeric rating score $\leq 3$ ) after 30 min. Secondary safety endpoints included serious adverse events and death at 30 days.

Results: The median baseline pain score was 7.0 in both groups. The primary endpoint occurred in $51.7 \%$ of the NOO-A group and $73.6 \%$ of the morphine group (absolute risk difference $-21.7 \%$; $95 \%$ confidence interval -29.6 to - 13.8). At 30 days, the rate of serious adverse events was 16.0 and $18.8 \%$ in the NOO-A and morphine groups respectively $(p=N S)$. The rate of death was 1.8\% (NOO-A group) and 3.8\% (morphine group) ( $p=N S$ ).

Conclusion: Analgesia provided by NOO-A was inferior to morphine at $30 \mathrm{~min}$ in patients with acute STEMI in the prehospital setting. Rates of serious adverse events did not differ between groups.
\end{abstract}

Trial registration: ClinicalTrials.gov: NCT02198378.

Keywords: ST-segment elevation myocardial infarction, Analgesia, prehospital

\footnotetext{
* Correspondence: charpentier.s@chu-toulouse.fr

Previous presentation: the results were presented at the European Society of

Cardiology congress in August 2018 in Munich (Germany).

'Emergency Department, Toulouse University Hospital, INSERM UMR 1027,

University Toulouse III Paul Sabatier, Toulouse, France

${ }^{2}$ Emergency Department, Rangueil University Hospital, 1 Av. Jean Poulhès,

31059 Toulouse, France

Full list of author information is available at the end of the article
}

C C The Author(s). 2020 Open Access This article is licensed under a Creative Commons Attribution 4.0 International License, which permits use, sharing, adaptation, distribution and reproduction in any medium or format, as long as you give appropriate credit to the original author(s) and the source, provide a link to the Creative Commons licence, and indicate if changes were made. The images or other third party material in this article are included in the article's Creative Commons licence, unless indicated otherwise in a credit line to the material. If material is not included in the article's Creative Commons licence and your intended use is not permitted by statutory regulation or exceeds the permitted use, you will need to obtain permission directly from the copyright holder. To view a copy of this licence, visit http://creativecommons.org/licenses/by/4.0/. The Creative Commons Public Domain Dedication waiver (http://creativecommons.org/publicdomain/zero/1.0/) applies to the data made available in this article, unless otherwise stated in a credit line to the data. 


\section{Background}

Pain can be particularly intense in ST-segment elevation myocardial infarction (STEMI), leading to tachycardia, increased stress, higher workload of the heart and damaging effects on the myocardium [1]. Analgesia, administered as soon as possible after symptom onset, is therefore of paramount importance. Opioids (most commonly morphine) are recommended, [2] although their efficacy and safety have not been fully evaluated in randomized trials. Recently, the deleterious effect of morphine on inhibition of platelet reactivity in STEMI patients treated with $\mathrm{P}_{2} \mathrm{Y}_{12}$ inhibitors has been reported [3-6]. Studies have reported that morphine is associated with a delayed onset of action of oral antiplatelet drugs due to vomiting or delayed gastric emptying, which reduce the absorption of these drugs [4].

Nitrous oxide/oxygen gas as an equimolar mixture is widely used in emergency medicine and has been tested in acute myocardial infarction [7]. It acts by activating opioid neurons, leading to activation of the descending noradrenergic inhibitory pathways that inhibit nociception [8]. It has minor, rapidly reversible secondary effects and no reported haemodynamic effects [9]. Few studies in emergency medicine have compared Nitrous OxidOxygen to morphine with heterogenous results [10-12]. Acetaminophen is an effective and safe painkiller for emergency department patients [13]. It has been successfully used in multimodal analgesia especially postoperative analgesia [14]. Nitrous oxide/oxygen plus intravenous acetaminophen (NOO-A) could therefore be a suitable alternative to morphine. The association nitrous oxidoxygen plus intravenous acetaminophen was chosen for reasons of delay in action. NOO has a 3-5 min onset of action and intravenous acetaminophen reaches its peak concentration at the end of the 15-min infusion. Thereafter, the duration of action of acetaminophen is $6 \mathrm{~h}$ while the effect of nitrous oxid-oxygen stops $5 \mathrm{~min}$ after inhalation is stopped. Thus, Acetaminofen allowed continuing the pain management once $\mathrm{NOO}$ was stopped.

The aim of the SCADOL II study was to assess in patients with acute STEMI managed in the prehospital setting the non-inferiority in achieving analgesia at $30 \mathrm{~min}$ of an equimolar mixture of NOO plus intravenous acetaminophen compared with intravenous morphine. The secondary safety objectives were the rates of serious adverse events and death at 30 days.

\section{Methods}

\section{Study design}

SCADOL II was a multicenter, open-label, clusterrandomized, controlled, non-inferiority trial. Thirtyeight mobile intensive care unit centers were randomized (1:1) to perform analgesia with NOO-A or intravenous morphine. A computerized randomization process was used to generate the random allocation sequence and was carried out by the methodologist from the list provided by participating centers. The details of the SCADOL II investigators list is provided in Additional file 1: Appendix 1.

The study has complied with the Declaration of Helsinki, the locally appointed ethics committee has approved the research protocol and informed consent has been obtained from the subjects (or their legally authorized representative).

\section{Selection of participants}

Patients aged $\geq 18$ years with suspected STEMI managed by an emergency physician in a mobile intensive care unit were eligible if they had symptom duration of $<12$ $h$ and a pain intensity score, assessed on a numeric rating scale, $\geq 4$ (pain scale range $0-10$ ).

Exclusion criteria were severe haemodynamic, respiratory, or neurological failure; heart failure; known allergy or contraindication to morphine or nitrous oxide; morphine or nitrous oxide administration within previous 4 $\mathrm{h}$; incapacity to self-assess pain intensity on a numeric rating scale; legal guardianship; pregnancy; or air ambulance transport.

The reperfusion strategy (thrombolysis or angioplasty) was chosen by the emergency physicians according to guidelines [15]. To prevent delays in the performance of revascularization, centers were randomly allocated before the start of the study, using a cluster design, to the NOO-A or morphine group.

\section{Study procedures}

Analgesics were started by the emergency physician as soon as possible after enrollment. In the control group, morphine administration was titrated every 5 min according to pain intensity, assessed on a numeric rating scale: a $2 \mathrm{mg}$ bolus (or $1 \mathrm{mg}$, for body weight $<60 \mathrm{~kg}$ ) was given for a numeric rating scale score $4-5$; and a $3 \mathrm{mg}$ bolus (or $2 \mathrm{mg}$, for body weight $<60 \mathrm{~kg}$ ) for a score $\geq 6$ [16]. In the intervention group, nitrous oxide/oxygen was administered according to the marketing authorization and under supervision of the emergency physician, and was given for $\geq 30$ min (a minimum of $5 \mathrm{~min}$ is necessary to obtain an analgesic effect) [17]. Nitrous oxide/oxygen was combined with $1 \mathrm{~g}$ intravenous acetaminophen in the framework of a multimodal analgesia. After $15 \mathrm{~min}$ of use, if the pain was still intense (numeric rating scale $\geq 6$ ) the emergency physician could change the analgesic strategy and use morphine; such patients were considered treatment failures. After $30 \mathrm{~min}$ and until arrival at hospital, the emergency physician could change the strategy of analgesia. 


\section{Study endpoints}

The primary endpoint was the proportion of patients achieving pain relief with NOO-A (without any morphine administration) or morphine, defined as a pain intensity score on a numeric rating scale $\leq 3[12,18], 30$ min after starting analgesia [19].

Secondary endpoints included the rate of pre-specified adverse events in the two groups: respiratory depression, defined as a respiratory rate $<10$ cycles per minute, or a respiratory score of $\geq 1$ (see Additional file 1 , definitions); nausea; vomiting; sedation (measured by a Sedation Scale score) of $\geq 2$; dizziness; and pruritus.

Data on pain intensity, adverse events, and tolerance (heart rate, non-invasive arterial pressure, pulse oximetry) were collected at baseline, every $5 \mathrm{~min}$ up to 30 min after the start of analgesia, and at hospital arrival.

A 30-day safety analysis was done on incidence of serious adverse events and death occurring in the 30 days post-treatment.

\section{Study oversight}

The executive and steering committee oversaw the conduct of the trial and the data analysis. The trial was monitored by a clinical research assistant and the data management was done by a data manager independent of the steering committee. Statistical analysis were performed blinded to treatment allocation. Finally, we completed the CONSORT checklist (Additional file 1: Appendix 2).

\section{Statistical analysis}

We estimated that 684 patients and 38 mobile intensive care unit centers (19 clusters) were needed to assess the non-inferiority of NOO-A to achieve pain relief at 30 min, given an $80 \%$ expected proportion of pain achievement in the control group, a $10 \%$ non-inferiority margin, a $2.5 \%$ one-sided alpha error rate, $80 \%$ power, and the cluster design. Analysis were performed using SAS version 9.4 (SAS Institute Inc., Cary, NC, USA). A noninferiority margin was specified in the protocol as an absolute difference of $-10 \%$ in proportion. The outcome was deemed to be non-inferior if the lower limit of the 95\% two-sided confidence interval (CI) was greater than the non-inferiority margin. The main effect of NOO-A versus morphine on the primary endpoint was assessed using an unadjusted generalized estimating-equations model with an exchangeable covariance matrix to account for the clustering of patients within centers. The intracluster correlation coefficient was estimated by the correlation parameter of the exchangeable covariance matrix and by using linear mixed effect models with a random center effect and the treatment as fixed effect, as the intracluster correlation coefficient calculated ignoring potential treatment effects may be biased [20].
Additional analysis of the primary endpoint included an adjustment for potential risk factors associated with analgesia (baseline pain score, age, sex, and thrombolysis). Random imputations were performed on the basis of the observed values to replace missing pain scores at baseline. Finally, a pre-specified subgroup analysis was done in patients with a confirmed STEMI diagnosis.

The per-protocol population (i.e. patients evaluable for the primary endpoint without major protocol deviations) was used for the primary endpoint analysis, as recommended for a non-inferiority trial [21]. Secondary analysis were done in the intention-to-treat population (i.e. all patients who entered the study, with the exception of patients from prematurely closed centers); pain scores missing at $30 \mathrm{~min}$ were imputed by means of the lastobservation-carried-forward method. If no pain score was present after baseline, missing values were taken to indicate failure (i.e. a pain score at $30 \mathrm{~min}$ of $>3$ ).

In the safety analysis, the incidence of expected adverse events (i.e. sedation, respiratory depression, vomiting, nausea, pruritus, and dizziness), unexpected adverse events, and adverse events leading to treatment discontinuation in the $30 \mathrm{~min}$ following initiation of analgesia were computed. The proportions of patients with $\geq 1$ expected adverse event in the 30 first minutes, and of serious adverse events that occurred in the 30 days posttreatment, were compared between groups using a generalized estimating equation model, to account for clustering.

\section{Results}

\section{Study population}

Between November 2014 and December 2016, 38 centers were randomized to the NOO-A or the morphine strategy (19 in each arm). A total of 684 patients were enrolled and composed the intention-to-treat population (340 in the NOO-A group; 344 in the morphine group), all of whom received the study treatment.

The per-protocol population comprised 644 patients (315 in the NOO-A group; 329 in the morphine group) (Fig. 1). The patient characteristics were well balanced between groups (Table 1 and Additional file 1: Appendix Table 1). Median pain intensity was 7.0 (interquartile Q1 to Q3: 5.0 to 8.0).

\section{Efficacy}

Patients in the morphine group were more likely to achieve pain relief than those in the NOO-A group (Fig. 2): the primary endpoint was obtained respectively in $73.6 \%$ in the morphine group versus $51.7 \%$ of patients in the NOO-A group. The absolute risk difference was $-21.7 \%$ [95\% Confidence Interval (CI) -29.6 to -13.8 ; intracluster correlation coefficient 0.009975 ), which was below the non-inferiority margin of $-10 \%$ defined in the 


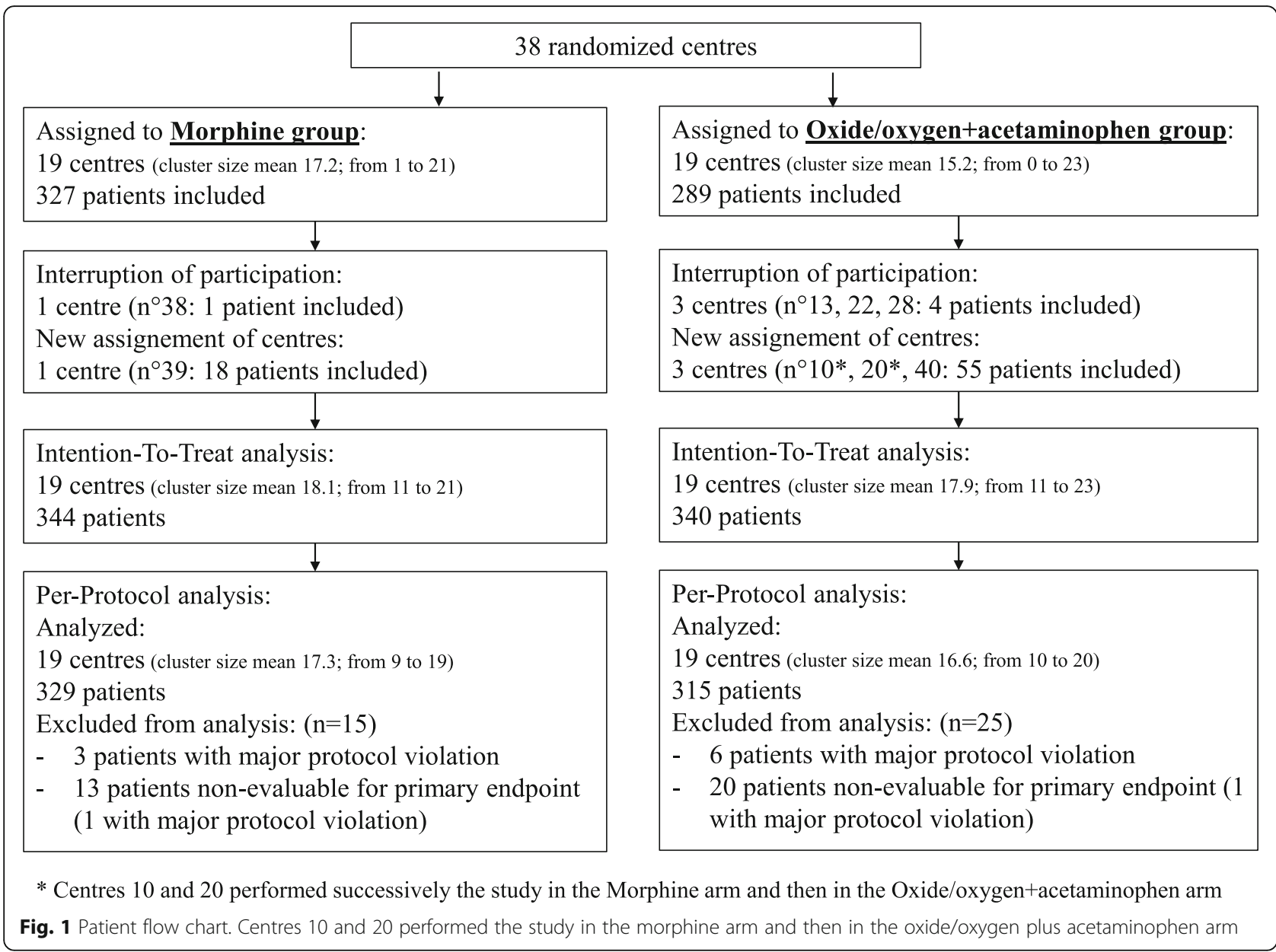

protocol (Fig. 3). Analysis of the primary endpoint in the intention-to-treat population showed the same effect (absolute risk difference -0.217 ; 95\% CI -0.297 to 0.136; intracluster correlation coefficient 0.01518).

A similar observation (more likely to achieve pain relief with morphine) was made in the subgroup of patients with a confirmed diagnosis of STEMI (NOO-A: 144 [50.3\%] vs. morphine: 218 [71.7\%]), with an absolute risk difference of -0.21 [95\% CI -0.29 to -0.13 ).

Analysis of the primary endpoint in the per-protocol population, adjusted for potential risk factors, showed that better relief of chest pain was associated with morphine treatment, lower pain score at baseline and increasing age (Table 2).

\section{Safety}

The percentage of patients with a predefined adverse event occurring within $30 \mathrm{~min}$ of starting analgesia was similar in the two study groups $(13.2 \%$ with NOO-A and $10.2 \%$ with morphine).

(Table 3) (absolute risk difference of 0.032 [95\% CI 0.01 to 0.07$] ; P=0.14)$. The most frequent expected adverse event was vomiting $(5.0 \%$ with NOO-A and $4.7 \%$ with morphine).

The percentage of patients with an unexpected (not predefined) adverse event within $30 \mathrm{~min}$ of starting analgesia was $6.2 \%$ with NOO-A and $3.5 \%$ with morphine, the most frequent being ventricular fibrillation $(1.2 \%$ with NOO-A and $0.9 \%$ with morphine). The rate of adverse events that led to treatment interruption within the first 30 min was $7.1 \%$ with NOO-A and $1.2 \%$ with morphine, the most frequent being vomiting $(2.9 \%$ with NOO-A and $0.3 \%$ with morphine).

The incidence of serious adverse event in the 30 days following inclusion was $18.8 \%$ with NOO-A and $16.0 \%$ with morphine (Table 3) (absolute risk difference of 0.033 [ $95 \% \mathrm{CI}-0.030$ to 0.096 ]; $P=0.3$ ). The most frequent were ventricular tachycardia (3.8\%), ventricular fibrillation (1.9\%), cardiogenic shock (1.8\%), and heart failure (1.1\%). Most cases of ventricular tachycardia (21 out of 26) were observed in the NOO-A group.

Nineteen patients died during the 30 days following inclusion (Additional file 1: Appendix Table 2), 6 in the NOO-A group (1.8\%) and 13 in the morphine group 
Table 1 Characteristics of patients in the per-protocol population

\begin{tabular}{|c|c|c|}
\hline & $\begin{array}{l}\text { Nitrous oxide/oxygen } \\
\text { plus acetaminophen } \\
(n=315)\end{array}$ & $\begin{array}{l}\text { Morphine } \\
(n=329)\end{array}$ \\
\hline Age, years, mean $\pm S D$ & $61.9 \pm 13.7$ & $62.1 \pm 13.0$ \\
\hline Male sex, $n(\%)$ & $255(81.0)$ & $249(75.7)$ \\
\hline Body mass index ${ }^{a} \mathrm{~kg} / \mathrm{m}^{2}$ & $n=296$ & $n=307$ \\
\hline Median (Q1; Q3) & $25.8(23.7 ; 28.1)$ & $\begin{array}{l}26.0(23.9 ; \\
29.0)\end{array}$ \\
\hline$\geq 30 \mathrm{~kg} / \mathrm{m}^{2}, n(\%)$ & $42(14.2)$ & $58(18.9)$ \\
\hline Smokers, n/N (\%) & $147 / 314(46.8)$ & $150 / 321(46.7)$ \\
\hline Diabetes, ${ }^{b}$ n/N (\%) & $36 / 312(11.5)$ & $38 / 323(11.8)$ \\
\hline Hypertension, ${ }^{b}$ n/N (\%) & $131 / 312(42.0)$ & $119 / 318(37.4)$ \\
\hline $\begin{array}{l}\text { Hypercholesterolaemia, } \\
n / N(\%)\end{array}$ & $82 / 305(26.9)$ & $95 / 315(30.2)$ \\
\hline $\begin{array}{l}\text { Family history of cardiovascular } \\
\text { disease, } n / N(\%)\end{array}$ & $94 / 274(34.3)$ & 87/278 (31.3) \\
\hline $\begin{array}{l}\text { Previous coronary artery disease, } \\
n / N(\%)\end{array}$ & $55 / 311(17.7)$ & $56 / 324(17.3)$ \\
\hline Thrombolysis, n (\%) & $30(9.5)$ & $46(14.0)$ \\
\hline Decision of angioplasty, $n$ (\%) & $296(94.0)$ & $308(93.6)$ \\
\hline \multicolumn{3}{|l|}{ Treatments at baseline, $n(\%)$} \\
\hline Aspirin & $313(99.4)$ & $323(98.2)$ \\
\hline $\begin{array}{l}\text { Other antiplatelet (clopidogrel, } \\
\text { ticagrelor or prasugrel) }\end{array}$ & $301(95.6)$ & $314(95.4)$ \\
\hline Clopidogrel & $54(17.1)$ & $56(17.0)$ \\
\hline Ticagrelor & $168(53.3)$ & $145(44.1)$ \\
\hline Prasugrel & $81(25.7)$ & $116(35.3)$ \\
\hline Heparin & $136(43.2)$ & $163(49.5)$ \\
\hline $\begin{array}{l}\text { Low-molecular-weight } \\
\text { heparin }\end{array}$ & $159(50.5)$ & $124(37.7)$ \\
\hline Bivalirudin & $21(6.7)$ & $32(9.7)$ \\
\hline $\begin{array}{l}\text { Anticoagulant (heparin, low- } \\
\text { molecular-weight heparin } \\
\text { or bivalirudin) }\end{array}$ & $307(97.5)$ & $318(96.7)$ \\
\hline Beta-blocker & $4(1.3)$ & 0 \\
\hline Glycoprotein Ilb/IIla inhibitor & $6(1.9)$ & $9(2.7)$ \\
\hline Anxiolytic & $2(0.6)$ & $10(3.0)$ \\
\hline $\begin{array}{l}\text { Other treatment (administered } \\
\text { in mobile intensive care unit) }\end{array}$ & 47 (14.9) & $69(21.0)$ \\
\hline $\begin{array}{l}\text { Delay between chest pain and } \\
\text { study treatment start, minutes }\end{array}$ & $n=314$ & $n=329$ \\
\hline Median (Q1; Q3) & $91.0(65.0 ; 161.0)$ & $\begin{array}{l}100.0(62.0 ; \\
167.0)\end{array}$ \\
\hline $\begin{array}{l}\text { Pain score on numeric rating } \\
\text { scale at study treatment start }\end{array}$ & $n=314$ & $n=328$ \\
\hline Median (Q1; Q3) & $7.0(5.0 ; 8.0)$ & $7.0(5.0 ; 8.0)$ \\
\hline
\end{tabular}

$Q$ quartile, $S D$ Standard deviation

${ }^{\mathrm{a}}$ Body mass index is the weight in kilograms divided by the square of the height in meters

${ }^{\mathrm{b}}$ Treated
$(3.8 \%)$. None of the deaths were judged to be directly related to the study treatment.

\section{Limitations}

The study has several limitations. Firstly, the low rate of events in both groups made the study underpowered to detect a clinically relevant difference in safety endpoints. It is a common drawback encountered in STEMI studies with prehospital recruitment that select lower risk patients. It does not by itself alter the conclusions of our study. Secondly, even if the cluster randomization provided a pragmatic comparison of chest pain control strategies in the prehospital setting, randomization takes place before consent to participate and individual recruitment. We tried to limit selection bias by a strict monitoring of the study and the rigorous recording of selection criteria. Of note, cluster randomization allowed for the NOO-A treatment to be better included in MICUs routine care. Influence of selection bias cannot be completely ruled out. Thirdly, participants, physicians and patients, were not blinded to the fact that they were receiving morphine or NOO-A. It is therefore possible that a measure reported by patients such as the numeric rating score be influenced by physicians' pre-existing convictions. From a practical perspective - a doubleblind study would have necessitated the transport and management of two gas cylinders in addition to the standard equipment. The open design simplified prehospital logistics and limited treatment delay.

\section{Discussion}

Contrary to our hypotheses, the main finding of our study are that 1) NOO-A was actually inferior to intravenous morphine for the reduction of pain at $30 \mathrm{~min}$ in patients with STEMI; 2) there were no more adverse events in the morphine group. Controlling pain at the acute phase of STEMI is challenging. Morphine has been used for years and the issues of its efficacy and its safety have been regularly raised [22, 23]. It has also been linked to a delayed onset of action of oral antiplatelet drugs $[4,5]$ Few alternatives to morphine have been studied in STEMI, and analgesics that are appropriate for the emergency setting (e.g. non-steroidal antiinflammatory drugs) are contraindicated [24-26].. The choice of NOO-A combination was especially relevant for use in STEMI. Nitrous oxide/oxygen has short onset of action. It has few unwanted effects [9]. Acetaminophen is an effective and safe painkiller for patients in the emergency department [13]. It was therefore intriguing that the NOO-A combination provided such a relatively low rate of pain relief with only half of the patients expressing a pain intensity less than 3 at $30 \mathrm{~min}$. In contrast, in the morphine group, the proportion of patients with adequate pain relief was high (73\%) and consistent 


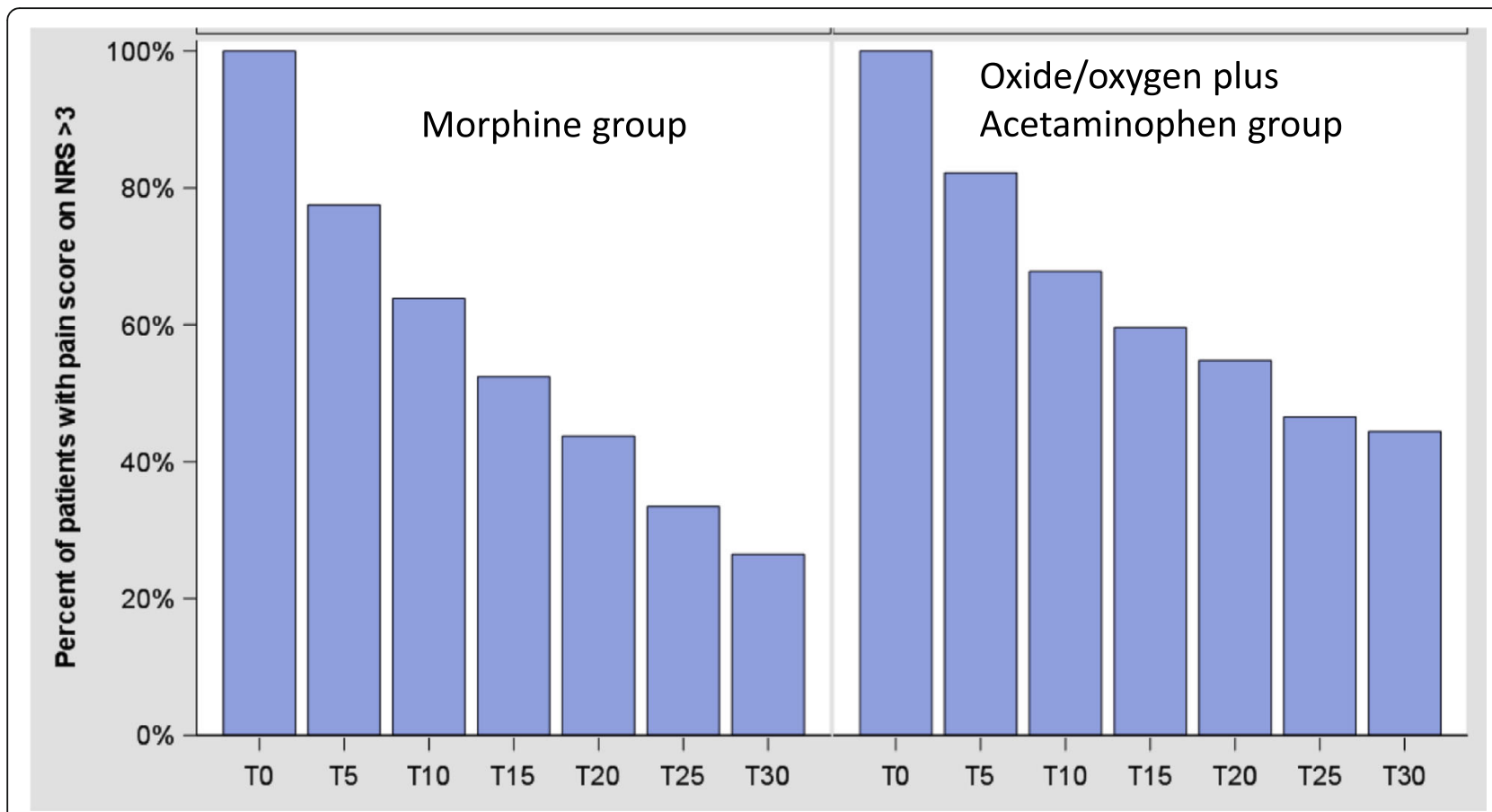

Fig. 2 Percent of patients with pain score (numeric rating scale [NRS]) $>3$. T, time in minutes

with other studies [27, 28]. Intravenous acetaminophen was administered at the recommended dose [16]. This dose proved at least as potent than morphine in patients with renal colitis [9]. It is possible that the NOO-A dosage was suboptimal in view of the high pain intensity of patients with STEMI. Importantly, both treatments were well tolerated. The rate of nausea or vomiting, events that were specifically followed per protocol, was low and did not differ between morphine and NOO-A groups. Morphine is usually considered as responsible of vomiting hence drug interactions, concerns for use in routine. Since NOO-A is not supposed to increase the rate of nausea and vomiting, it may be inferred that the role of morphine in causing nausea and vomiting in STEMI has been overstated. Other factors, especially parasympathetic effect in STEMI with an inferior location and pain

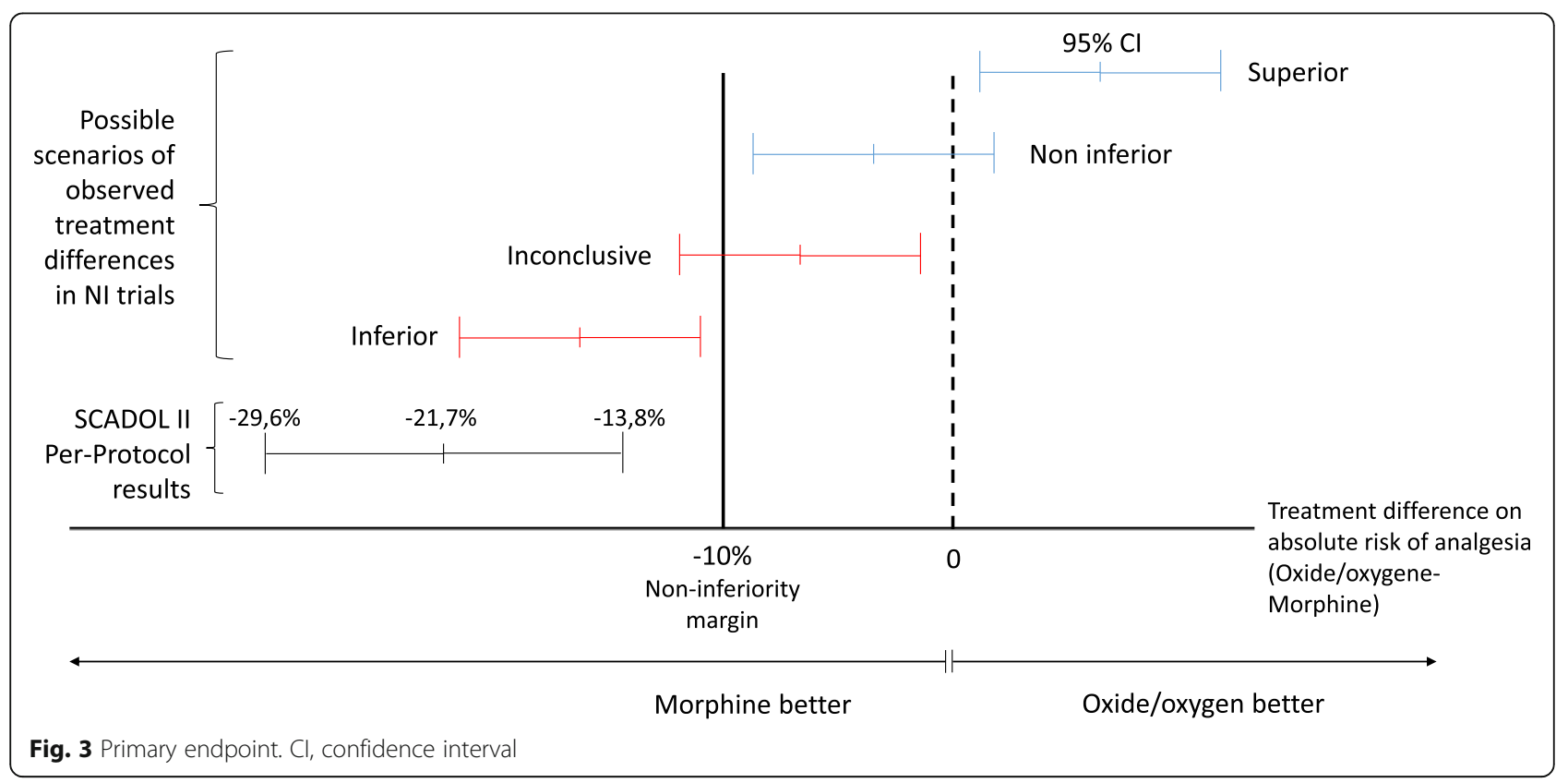


Table 2 NOO-A plus acetaminophen versus morphine: adjusted effect on primary endpoint estimated with estimating equations model

\begin{tabular}{lll}
\hline Parameter & OR (95\% Cl) & $P$-Value \\
\hline $\begin{array}{l}\text { Nitrous oxide/oxygen plus acetaminophen } \\
\text { (vs. morphine) }\end{array}$ & $0.32(0.21-0.49)$ & $<0.0001$ \\
$\begin{array}{l}\text { Pain score on numeric rating score at } \\
\text { baseline (per 1-point increase) }\end{array}$ & $0.58(0.51-0.67)$ & $<0.0001$ \\
Age (per 1-year increase) & $1.02(1.00-1.03)$ & 0.017 \\
Thrombolysis (vs. none) & $0.96(0.51-1.79)$ & 0.89 \\
Male sex & $0.83(0.58-1.18)$ & 0.29 \\
\hline
\end{tabular}

NOO-A Nitrous oxide oxygen, $\mathrm{Cl}$ Confidence interval $O R$ Odds ratio

intensity may play a more important role. It is clinically relevant because vomiting and delayed gastric emptying have been incriminated in the delayed onset of action of oral antiplatelet drugs in patients treated with morphine [4].

Overall, the rate of adverse events was not significantly different between the NOO-A and the morphine groups. The number of deaths was numerically higher in the morphine group (3.8\% vs $1.8 \%$ at 1 month). This statistically non-significant higher death rate in the morphine group should not be an argument to renounce to this potent analgesic in STEMI patients. However, this

Table 3 Incidence of adverse events (intention-to-treat population)

\begin{tabular}{|c|c|c|}
\hline Event, $n(\%)$ & $\begin{array}{l}\text { Nitrous oxide/oxygen plus } \\
\text { acetaminophen }(n=340)\end{array}$ & $\begin{array}{l}\text { Morphine } \\
(n=344)\end{array}$ \\
\hline \multicolumn{3}{|c|}{ Adverse events in the 30 min after starting treatment } \\
\hline$\geq 1$ expected adverse event & $45(13.2)$ & $35(10.2)$ \\
\hline $\begin{array}{l}\text { Respiratory depression } \\
(<10 \text { cycles/min or } \\
\text { score } \geq \text { R } 1)\end{array}$ & $4(1.2)$ & $5(1.5)$ \\
\hline Nausea (without vomiting) & $11(3.2)$ & $8(2.3)$ \\
\hline Vomiting & $17(5.0)$ & $16(4.7)$ \\
\hline Sedation (score of $\geq 2$ ) & $15(4.4)$ & $7(2.0)$ \\
\hline Dizziness & $3(0.9)$ & $3(0.9)$ \\
\hline Pruritus & 0 & $3(0.9)$ \\
\hline $\begin{array}{l}\geq 1 \text { unexpected serious } \\
\text { adverse event }\end{array}$ & $21(6.2)$ & $12(3.5)$ \\
\hline $\begin{array}{l}\text { Adverse event that led to } \\
\text { treatment interruption }\end{array}$ & $24(7.1)$ & $4(1.2)$ \\
\hline $\begin{array}{l}\text { Serious adverse event in the } \\
30 \text { days after enrolment }\end{array}$ & $64(18.8)$ & $55(16.0)$ \\
\hline \multicolumn{3}{|c|}{ Adverse event occurring in $\geq 1 \%$ of patients } \\
\hline Ventricular tachycardia & $21(6.2)$ & $5(1.5)$ \\
\hline Ventricular fibrillation & $8(2.4)$ & $5(1.5)$ \\
\hline Cardiogenic shock & $5(1.5)$ & $7(2.0)$ \\
\hline Heart failure & $3(0.9)$ & $5(1.5)$ \\
\hline $\begin{array}{l}\text { Death in the } 30 \text { days after } \\
\text { enrolment }\end{array}$ & $6(1.8)$ & $13(3.8)$ \\
\hline
\end{tabular}

observation adds to the uncertainties surrounding the safety of morphine in ACS even if recent studies have not shown an increase in mortality in patients treated with morphine [5].

\section{Conclusion}

NOO-A was inferior to morphine analgesia at $30 \mathrm{~min}$ in patients with acute STEMI in the prehospital setting. Rates of adverse events were not significantly different between the two treatment groups. Because morphine appears to be such a potent agent of pain control in STEMI, a randomised study specifically addressing its safety is warranted.

\section{Supplementary information}

Supplementary information accompanies this paper at https://doi.org/10. 1186/s13049-020-00731-y.

Additional file 1: Appendix 1. SCADOL $\|$ Investigators list. Appendix 2. CONSORT 2010 checklist of information to include when reporting a randomised trial. Appendix 3. Definition of secondary outcomes. Appendix Table 1. Characteristics of Patients in the Intention-To-Treat Population. Appendix Table 2. Characteristics of Patients Who Died Within 30 Days.

\section{Abbreviations}

STEMI: ST-segment elevation myocardial infarction; NOO: Nitrous Oxide/ Oxygen; NOO-A: Nitrous oxide/oxygen plus intravenous Acetaminophen; MICU: Medical intensive care unit; ACS: Acute coronary syndrome

\section{Acknowledgements}

The authors acknowledge the sponsor, Toulouse University Hospital, the patients, physicians, and nurses who participated in the study; Elodie Tournay, who checked the statistical section; Christophe Morin, who performed the data management; and the research assistants Vanessa Houze Cerfon, Manon Hebrard, Caroline Peyrot, and Alexandra Seux. Sophie K. Rushton-Smith, PhD (MedLink Healthcare Communications, London) provided editorial support on the final version and was funded by the authors. This work was supported by a grant from the Clinical Research Hospital Program of the French Ministry of Health (PHRC 2013), the French Society of Emergency Medicine, and the French Society of Cardiology. SCADOL II Investigators list

Vincent Bounes, SAMU 31, Toulouse University Hospital, University Toulouse III Paul Sabatier, Toulouse, France

Claire Vallenet, SMUR Annemasse, Annemasse-Bonneville Hospital, Annemasse, France

Elise Robeley, SAMU 25, Jean Minjoz University Hospital, Besançon, France Frédéric Lapostolle, SAMU 93, UF Recherche-Enseignement-Qualité Université Paris 13, Sorbonne Paris Cité, Inserm U942 Hôpital Avicenne, AP-HP, Bobigny, France

Catherine Pradeau, SAMU 33, Pellegrin University Hospital, Bordeaux, France Patrice Serre, SMUR Bourg en Bresse, Flevriat, Bourg en Bresse Carols El Khoury, Emergency Department and RESCUe Network, Lucien Hussel Hospital, Vienne, France, Univ. Lyon, Claude Bernard Lyon 1 University, HESPER EA 7425, Lyon, France

Pascal Usseglio, SMUR Chambéry, Chambéry Hospital, Chambéry,France Eric Revue, SMUR Chartres, Chartres Hospital, Chartres, France

Christine Bregeaud, SMUR Chateauroux, Hospital Centre of Chateauroux, Chateauroux, France

Christine Lespiaucq, SAMU 63, Clermont-Ferrand University Hospital,

Clermont-Ferrand, France

Sonja Curac, SMUR Beaujon, Beaujon Hospital, Clichy, France Julie Jardon, SAMU 09, Val d'Ariège Hospital, Foix, France Pierre Arnaud Fort, SAMU 47, Agen Hospital,Agen, France Armelle Severin, SAMU 92, Poincaré University Hospital, Garches, France 
Guillaume Debaty, SAMU 38, Grenoble University Hospital, Grenoble France Anne-Sophie Lucas, SAMU 85, La-Roche-Sur-Yon Hospital, La-Roche-Sur-Yon, France

Bahram Chaybany, SAMU 59, Lilles University Hospital, Lilles, France Alexandre Gerard, SAMU 69, Edouard Herriot Univseristy Hospital, Lyon, France

Marc Fournier, SAMU 13, La Timone Univesity Hospital, Marseille, France Anais Bauer, SAMU 57, Metz University Hospital, Metz, France Mustapha Sebbane, SAMU 34, Montpelier University Hospital, Montpellier France

Tahar Chouihed, SAMU 54, Nancy University Hospital, Nancy, France Camille Machet, SAMU 44, Nantes University Hospital, Nantes, France Julie Labiau, SAMU 06, Nice University Hospital, Nice, France

Claire Broche, SMUR Lariboisière, Lariboisière Univesrity Hospital, Paris, France Céline Maisondieu, SMUR Pitié- Salpêtrière, Pitié- Salpêtrière University Hospital, Paris, France

Matthieu Marchetti, SAMU 86, Poitiers University Hospital, Poitiers, France Agnès Ricard-Hibon, Pole Emergency Department - SAMU - René Dubos Hospital, Pontoise, France

François-Xavier Ageron, Emergency Department, Centre Hospitalier Annecy Genevois, Annecy, France

Nicolas Bohrer, SAMU 97, Felix Guyon University Hospital, Saint Denis de la Réunion, France

Laurent Teillol, SMUR Saint Gaudens, Comminges Pyrennees Hospital, Saint Gaudens, France

Muriel Vergne, SAMU 83, Toulon Hospital, Toulon, France

Didier Dansou, SAMU 37, Tours University Hospital, Tours, France

Dominique Cailloce, SAMU 87, Limoges University Hospital, Limoges, France

David Sapir, SMUR Corbeil, Sud Francilien Hospital, Corbeil Essonnes, France

\section{Authors' contributions}

Sandrine Charpentier (SC), Eric Bonnefoy (EB), Vincent Bounes (VB), Agnès Ricard-Hibon (ARH), Michel Galinski (MG), Stéphane Manzo-Silbermann (SMS), Fréderic Lapostolle (FL), Louis Soulat (LS), Carlos El Khoury (CEK) and Meyer Elbaz (ME) conceived the study, designed the trial, and obtained research funding. SC and EB supervised the conduct of the trial and data collection. SC, VB, FL, ARH, LS, CEK, Delphine Bregeaud (DB) and Alexandre Gerard (AG) undertook recruitment of participating centers and patients and managed the data, including quality control. Vanina Bongard (VB) provided statistical advice on study design and analyzed the data; SC chaired the data oversight committee. SC drafted the manuscript, and all authors contributed substantially to its revision. SC takes responsibility for the paper as a whole. The authors read and approved the final manuscript.

\section{Funding}

This work was supported by the French Society of Emergency Medicine and the French Society of Cardiology and obtained a grant from the Clinical Research Hospital Program of the French Ministry of Health (PHRC 2013). Data management and analysis were the responsibility of the coordinating center and were independent of the sponsor. The funding organization had no involvement in the design of the study, the interpretation of the data and the decision to approve publication of the finished manuscript.

\section{Availability of data and materials}

The datasets used and/or analysed during the current study are available from the corresponding author on reasonable request.

\section{Ethics approval and consent to participate}

The study has complied with the Declaration of Helsinki, the locally appointed ethics committee (Comité de Protection des Personnes Sud-Ouest et Outre-Mer II, 2-14-12) has approved the research protocol and informed consent has been obtained from the subjects (or their legally authorized representative). It was registered at Clinical trial, number NCT02198378. All patients provided written consent before participation.

\section{Consent for publication}

Not applicable.

\section{Competing interests}

The authors declare that they have no competing interests.

\section{Author details}

'Emergency Department, Toulouse University Hospital, INSERM UMR 1027, University Toulouse III Paul Sabatier, Toulouse, France. '²mergency Department, Rangueil University Hospital, 1 Av. Jean Poulhès, 31059 Toulouse, France. ${ }^{3}$ Emergency Department - SAMU 33, CHU de Bordeaux; INSERM U1219 - Injury Epidemiology Transport Occupation" team, University Bordeaux II, 33000 Bordeaux, France. ${ }^{4}$ SAMU31, Toulouse University Hospital; University Toulouse III Paul Sabatier, Toulouse, France. ${ }^{5}$ Pôle Emergency Department, SAMU - Centre Hospitalier René Dubos Pontoise, 95300 Pontoise, France. ${ }^{6}$ Emergency Department and RESCUe Network, Lucien Hussel Hospital, Vienne, France. ${ }^{7}$ Univ. Lyon, Claude Bernard Lyon 1 University, HESPER EA, 7425 Lyon, France. ${ }^{8}$ Department of Cardiology, Rangueil University Hospital, Toulouse, France. ${ }^{9}$ Emergency Department, Centre Hospitalier Annecy Genevois, Annecy, France. ${ }^{10}$ Cardiology department, Lariboisire Hospital, APHP, Paris, France. ${ }^{11}$ Paris VII University UMRS 942, Paris, France. ${ }^{12}$ SAMU 35 SMUR Urgences adultes, Centre Hospitalier Universitaire Rennes, Université Rennes 1, Rennes, France. ${ }^{13}$ SAMU 93 - UF Recherche-Enseignement-Qualité Université Paris 13, Sorbonne Paris Cité, Inserm U942 Hôpital Avicenne, AP-HP, 125, rue de Stalingrad, 93009 Bobigny, France. ${ }^{14}$ Hospices Civils de Lyon SAMU 69 - Hôpital Édouard HERRIOT 5, place d'Arsonval, 69437 LYON Cedex 03, France. ${ }^{15}$ Hospital Centre of Chateauroux, Chateauroux, France. ${ }^{16}$ Department of Epidemiology, Centre Hospitalier Universitaire de Toulouse, Toulouse, France. ${ }^{17}$ Department of Public Health, Université Toulouse 3; UMR 1027 INSERM - Université Toulouse 3, Toulouse, France. ${ }^{18}$ Hôpital cardiologique Louis-Pradel, 69500 Lyon, France. ${ }^{19}$ Université Lyon-1, 69100 Lyon, France.

\section{Received: 17 March 2020 Accepted: 28 April 2020}

\section{Published online: 12 May 2020}

\section{References}

1. Leach A, Fisher M. Myocardial ischaemia and cardiac pain - a mysterious relationship. Br J Pain. 2013;7:23-30

2. Ibanez B, James S, Agewall S, et al. 2017 ESC guidelines for the management of acute myocardial infarction in patients presenting with STsegment elevation: the task force for the management of acute myocardial infarction in patients presenting with ST-segment elevation of the European Society of Cardiology (ESC). Eur Heart J. 2018;39:119-77.

3. Montalescot G, van't Hof AW, Lapostolle F, et al. Prehospital ticagrelor in STsegment elevation myocardial infarction. N Engl J Med. 2014;371:1016-27.

4. Kubica J, Adamski P, Ostrowska M, et al. Morphine delays and attenuates ticagrelor exposure and action in patients with myocardial infarction: the randomized, double-blind, placebo-controlled IMPRESSION trial. Eur Heart J. 2016:37:245-52.

5. Bellandi B, Zocchi C, Xanthopoulou I, et al. Morphine use and myocardial reperfusion in patients with acute myocardial infarction treated with primary PCI. Int J Cardiol. 2016;221:567-71.

6. Duartes GS, Nunes-Ferreira A, Rodrigues FB, et al. Morphine in acute coronary syndrome : systematic review and meta-analysis. BMJ Open. 2019; 9(3):e025232

7. Kerr F, Brown MG, Irving JB, et al. A double-blind trial of patient-controlled nitrous-oxide/oxygen analgesia in myocardial infarction. Lancet. 1975;1: 1397-400.

8. Orii R, Ohashi $Y$, Halder $S$, et al. GABAergic interneurons at supraspinal and spinal levels differentially modulate the antinociceptive effect of nitrous oxide in Fischer rats. Anesthesiology. 2003;98:1223-30.

9. Onody P, Gil P, Hennequin M. Safety of inhalation of a $50 \%$ nitrous oxide/ oxygen premix: a prospective survey of 35828 administrations. Drug Saf. 2006:29:633-40.

10. Volmanen P, Akural E, Raudaskoski T, et al. Comparison of remifentanil and nitrous oxide in labour analgesia. Acta Anaesthesiol Scand. 2005;49:453-8.

11. Kariman H, Majidi A, Amiri A, et al. Nitrous oxide/oxygen compared with fentanyl in reducing pain among adults with isolated extremity trauma: a randomized trial. EMA. 2011;23:761-8.

12. Paris $A$, Horvath $R$, Basset $P$, et al. Nitrous oxide-oxygen mixture during care of bedsores and painful ulcers in the elderly : a randomized, crossover, open-label pilot study. J Pain Symptom Manag. 2008;35:171-6.

13. Bektas F, Eken C, Karadeniz O, et al. Intravenous paracetamol or morphine for the treatment of renal colic: a randomized, placebo-controlled trial. Ann Emerg Med. 2009;54:568-74. 
14. Kehlet H. Multimodal approach to control postoperative pathophysiology and rehabilitation. Br J Anaesth. 1997;78:606-17.

15. The Task Force on the management of ST-segment elevation acute myocardial infarction of the European Society of Cardiology (ESC). ESC guidelines for the management of acute myocardial infarction in patients presenting with ST-segment elevation. Eur Heart J. 2012;33:2569-619.

16. Ricard-Hibon A, Bounes V. Sedation and analgesia in emergency structure. Which sedation and analgesia for the spontaneously breathing patient in emergency units? Ann Fr Anesth Reanim. 2012;31:295-312.

17. Maslekar S, Balaji P, Gardiner A, et al. Randomized controlled trial of patientcontrolled sedation for colonoscopy: entonox vs modified patientmaintained target-controlled proporfol. Color Dis. 2010;13:48-57.

18. Ohnhaus EE, Adler R. Methodological problems in the measurement of pain: a comparison between the verbal rating scale and the visual analog scale. Pain. 1975;1:379-84.

19. Lvovschi V, Aubrun F, Bonnet $P$, et al. Intravenous morphine titration to treat severe pain in the ED. Am J Emerg Med. 2008;26:676-82.

20. Giraudeau B. Model mis-specification and overestimation of the intraclass correlation coefficient in cluster randomized trials. Stat Med. 2006;25:957-64

21. Piaggio G, Elbourne DR, Pocock SJ, et al. Reporting of noninferiority and equivalence randomized trials: extension of the CONSORT 2010 statement. JAMA. 2012:308:2594-604.

22. Everts B, Karlson BW, Herlitz J, Hedner T. Morphine use and pharmacokinetics in patients with chest pain due to suspected or definite acute myocardial infarction. Eur J Pain. 1998;2:115-25.

23. Meine TJ, Roe MT, Chen AY, et al. Association of intravenous morphine use and outcomes in acute coronary syndromes: results from the CRUSADE quality improvement initiative. Am Heart J. 2005;149:1043-9.

24. Parodi G. Editor's choice-chest pain relief in patients with acute myocardial infarction. Eur Heart J Acute Cardiovasc Care. 2016;5:277-81.

25. Gislason GH, Jacobsen S, Rasmussen JN, et al. Risk of death or reinfarction associated with the use of selective cyclooxygenase-2 inhibitors and nonselective nonsteroidal antiinflammatory drugs after acute myocardial infarction. Circulation. 2006;113:2906-13.

26. Gardtman M, Dellborg M, Brunnhage C, et al. Effect of intravenous metoprolol before hospital admission on chest pain in suspected acute myocardial infarction. Am Heart J. 1999:137:821-9.

27. Hoff HR, Hotz MM, Sperber RJ, et al. Analgesia in myocardial infarction: double-blind comparison of Piminodine and morphine. Am J Med Sci. 1965: 249:495-8.

28. Zedigh C, Alho A, Hammar E, et al. Aspects on the intensity and the relief of pain in the prehospital phase of acute coronary syndrome: experiences from a randomized clinical trial. Coron Artery Dis. 2010;21:113-20.

\section{Publisher's Note}

Springer Nature remains neutral with regard to jurisdictional claims in published maps and institutional affiliations.

Ready to submit your research? Choose BMC and benefit from:

- fast, convenient online submission

- thorough peer review by experienced researchers in your field

- rapid publication on acceptance

- support for research data, including large and complex data types

- gold Open Access which fosters wider collaboration and increased citations

- maximum visibility for your research: over $100 \mathrm{M}$ website views per year

At BMC, research is always in progress.

Learn more biomedcentral.com/submissions 